\title{
La Vraie Forme d'un Arbre
}

\author{
J.Bétréma \& A.Zvonkin \\ LaBRI, Université Bordeaux I \\ 351, cours de la Libération, F33405 Talence \\ Unité associèe CNRS 1304
}

\section{Introduction}

A.Grothendieck a observé, dans son mémoire [4], que pour toute carte combinatoire, il existe une surface de Riemann compacte de même genre, sur laquelle la carte peut être dessinée de façon canonique (à un automorphisme de cette surface près): c'est la théorie des dessins d'enfants.

Cette théorie implique, comme cas particulier, que tout arbre plan possède un dessin canonique, à une similitude directe près; de plus, elle fait apparaître une relation de conjugaison entre arbres plans, qui ne se réduit à aucune autre relation connue aujourd'hui, et une action du groupe de Galois sur ces arbres, avec pour orbites les classes de conjugaison.

Le calcul effectif des dessins, des classes de conjugaison, et des groupes de Galois associés, n'est pas facile, même pour de petits arbres, ce qui gêne la formulation et le test de conjectures. Le premier catalogue exhaustif pour les arbres avec au plus 8 arêtes, a été réalisé, à notre connaissance, par les auteurs au printemps 1992 [3]; son volume interdit de le reproduire ici; cet article présente les méthodes employées pour sa réalisation, illustrées sur le cas des arbres à 6 arêtes.

La section 2 donne les définitions et théorèmes fondamentaux de la théorie des dessins d'enfants appliquée aux arbres. La section 3 expose les principes des calculs, illustrés section 4; les Figs. 3 et 4 donnent les dessins canoniques des 14 arbres à 6 arêtes. La section finale résume l'état de l'art et les perspectives dans ce domaine de recherche.

Note sur les logiciels employés: nous avons utilisé Maple pour les calculs euxmêmes. Il est vite apparu impossible, dans notre cas, de réaliser des dessins de qualité à partir des résultats produits par Maple, en utilisant les outils de dessin standard, comme la fonction plot; nous avons donc créé un logiciel de dessin spécialisé pour réaliser les dessins sous X-Windows, à l'aide de la boîte à outils Xview, pour OpenLook; les fichiers produits par Maple sont d'abord traités par un analyseur lexicographique simple, écrit en lex.

\section{$2 \quad$ Arbres et Dessins d'enfants}

\subsection{Arbres Plans}

Dans cet article, un arbre désignera un graphe connexe sans circuit, un arbre plan un arbre dessiné (plongé) dans le plan. Le dessin d'un arbre plan définit, 
pour chaque sommet de l'arbre, une permutation circulaire sur les sommets adjacents; un isomorphisme d'arbres plans est un isomorphisme d'arbres qui conserve ces permutations circulaires, et donc l'orientation; une classe d'isomorphisme d'arbres plans est appelée arbre plan combinatoire.

Pour fixer les idées, la Fig.1 présente les trois plus petits arbres ( 6 arêtes) possédant chacun deux dessins (numérotés $a$ et $b$ ) non isomorphes.

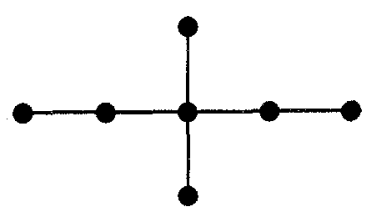

1.a

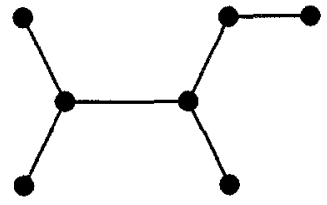

2.a

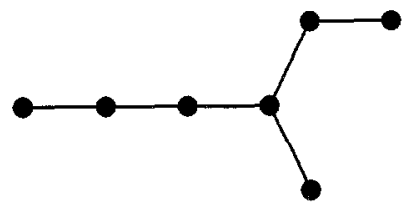

3.a

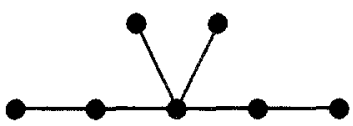

1.b

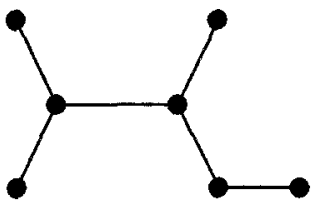

2.b

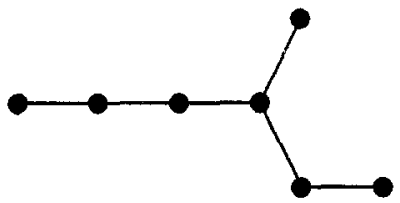

3.b

Fig. 1. Arbres plans non isomorphes

Les arbres plans combinatoires ont été énumérés dans [5], en utilisant le théorème de Pólya; la série énumératrice selon le nombre d'arêtes débute par:

$$
1+x+x^{2}+2 x^{3}+3 x^{4}+6 x^{5}+14 x^{6}+34 x^{7}+95 x^{8}+280 x^{9}+854 x^{10} \ldots
$$

à comparer avec celle des arbres:

$$
1+x+x^{2}+2 x^{3}+3 x^{4}+6 x^{5}+11 x^{6}+23 x^{7}+47 x^{8}+106 x^{9}+235 x^{10} \ldots
$$

\subsection{Polynômes de Shabat}

Considérons maintenant un polynôme à coefficients complexes $A(z)$, et soit $a$ une racine (complexe) de $A$; l'image réciproque par $A$ du segment réel $\left[\begin{array}{ll}0 & \epsilon\end{array}\right]$, pour $\epsilon$ petit, a l'aspect suivant:

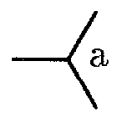


avec un nombre de brins égal à la multiplicité de la racine a (ici 3). De même, soit $b$ tel que $A(b)=1$; l'image réciproque par $A$ du segment $[1-\epsilon, 1]$ a un aspect similaire, avec un nombre de brins égal à la multiplicité de la racine $b$ pour le polynôme $B(z)=A(z)-1$.

Un point $u$ tel que $A^{\prime}(u)=0$ est appelé point critique; $v=A(u)$ est une valeur critique, et l'ordre du point critique $u$ est égal par définition à son ordre de multiplicité en tant que racine du polynôme $A-v$; l'ordre de $u$ est aussi la plus petite valeur de l'entier $k$ tel que la dérivée $k^{\text {ème }} A^{(k)}(u)$ soit non nulle. Notons $\Gamma$ l'image réciproque par A du segment [ll 1$]$ :

$$
\Gamma=A^{-1}\left(\left[\begin{array}{ll}
0 & 1
\end{array}\right]\right) \text {. }
$$

Si l'on suppose maintenant que les seules valeurs critiques de $A$ sont 0 et 1 (autrement dit: tout point critique est racine de $A$ ou de $A-1$ ), $\Gamma$ est constitué d'une collection de segments (courbes en général) qui relient les racines de $A$ aux racines de $A-1$, sans se couper (car deux segments ne peuvent se couper qu'en un point critique). $\Gamma$ est donc le dessin d'un graphe planaire, dont les sommets sont alternativement racines de $A$ et de $A-1$, avec pour degrés les ordres de multiplicité de ces racines. On prouvera section 3 que $\Gamma$ est en fait un arbre plan.

Dans la suite on appellera noirs (resp. blancs) les sommets correspondant aux racines de $A$ (resp. $A-1$ ). La théorie des dessins d'enfants, appliquée aux arbres, fournit la réciproque suivante:

Théorème 1. Pour tout arbre plan $\Gamma$, il existe un polynôme $A(z)$, admettant pour valeurs critiques au plus 0 et 1 , et tel que l'image réciproque du segment $\left[\begin{array}{l}0 \\ 1]\end{array}\right]$ par $A$ soit isomorphe à $\Gamma$; de plus $A$ est unique, aux deux transformations suivantes près:

(i) changer $z$ en $a z+b$ (similitude directe)

(ii) changer $A$ en $1-A$ (échange des couleurs des sommets).

Nous ne connaissons pas de preuve élémentaire de ce théorème. Suite aux travaux de G.Shabat $[1,6]$, nous appellerons dans la suite polynôme de Shabat d'un arbre plan $\Gamma$, tout polynôme $A$ satisfaisant aux conditions du Théorème 1; l'image réciproque du segment [0 1] par $A$ est appelée dessin canonique de $\Gamma$. La Fig. 2 montre ce dessin pour l'arbre 2.a de la Fig.1; les sections 3 et 4 expliquent comment obtenir ce dessin. Le polynôme de Shabat et le dessin canonique de $\Gamma$ sont unniques à similitude directe, et échange des couleurs, près.

Note: Les seuls polynômes admettant pour unique valeur critique 0 ou 1 sont $z^{n}$ et $z^{n}-1$; tous les autres dans cet article admettent donc deux valeurs critiques, et deux seulement.

\subsection{Arbres Conjugués et Action du Groupe de Galois}

Le calcul des coefficients d'un polynôme de Shabat se ramène, comme on le verra sections 3 et 4, à la résolution d'un système d'équations algébriques à coefficients entiers; les coefficients d'un polynôme de Shabat peuvent donc être choisis dans un corps de nombres algébriques $K$. Comme un polynôme de Shabat est défini au changement de variable $z \rightarrow a z+b$ près, $K$ n'est pas unique; mais on a: 
Théorème 2. Soit $\Gamma$ un arbre plan; il existe un corps algébrique minimal $K$ de définition des coefficients d'un polynôme de Shabat associé à $\Gamma$.

Le corps $K$ défini par ce théorème est appelé corps de définition de $\Gamma$; il est impossible de donner dans cet article une preuve rigoureuse du Théorème 2 , mais dans les calculs de la section 4, le corps de définition du dessin apparaît très naturellement.

Si $K$ est le corps des rationnels $\mathbf{Q}$, c'est à dire si $\Gamma$ possède un polynôme de Shabat à coefficients rationnels, l'arbre $\Gamma$ lui-même sera dit rationnel-- le risque de confusion avec l'emploi de ce mot dans le contexte de la théorie des langages d'arbres, semble minime.

Si le corps de définition est une extension algébrique propre de $\mathbf{Q}$, le calcul du polynôme de Shabat fait apparaître une famille de dessins conjugués, attachés aux diverses solutions de la même équation algébrique; et le groupe de Galois, qui agit sur ces solutions, agit donc sur ces dessins.

La théorie des dessins d'enfants associe aussi un corps de définition à toute carte combinatoire, bien que l'analogue du Théorème 2 ne soit plus valable sous la même forme dans le cas général. G.Belyi a démontré dans [2] que, pour tout corps de nombres $K$, il existe une carte dont $K$ est le corps de définition; on ignore actuellement s'il en est de même en se restreignant aux arbres.

\section{Calcul du Polynôme de Shabat}

\subsection{Polynômes avec Deux Valeurs Critiques}

Donnons d'abord la preuve, annoncée section 2.2, de la proposition dont le Théorème 1 constitue la réciproque:

Proposition 1. Soit $A$ un polynôme non constant admettant pour seules valeurs critiques 0 et 1 ; autrement dit:

$$
A^{\prime}(z)=0 \text { implique } A(z)=0 \text { ou } A(z)=1 .
$$

Alors $\Gamma=A^{-1}\left(\left[\begin{array}{ll}0 & 1\end{array}\right]\right)$ est un arbre plan.

Preuve: $\Gamma$ ne peut comporter de circuit, car la partie imaginaire d'une fonction polynomiale est harmonique, et ne peut donc être nulle sur un circuit sans être nulle dans toute la région bornée par ce circuit, ce qui est impossible pour un polynôme non constant. Donc $\Gamma$ est un arbre ou une forêt; pour prouver que $\Gamma$ est connexe, il suffit de compter arêtes et sommets.

Soit $n$ le degré de $A$ : c'est aussi le nombre d'arêtes de $\Gamma$; soit $p$ (resp. q) le nombre de racines distinctes de $A$ (resp. $A-1$ ), et $\alpha_{1}, \alpha_{2}, \ldots, \alpha_{p}$ (resp. $\beta_{1}, \beta_{2}, \ldots, \beta_{q}$ ) leurs multiplicités. On a évidemment:

$$
\sum_{i=1}^{p} \alpha_{i}=\sum_{j=1}^{q} \beta_{j}=n .
$$


Si $\alpha_{i}>1$, la racine correspondante est aussi racine d'ordre $\alpha_{i}-1$ de $A^{\prime}$; idem pour les $\beta_{j}$; comme par hypothèse toutes les racines de $A^{\prime}$ sont des racines de $A$ ou $A-1$, on a:

$$
\sum_{i=1}^{p}\left(\alpha_{i}-1\right)+\sum_{j=1}^{q}\left(\beta_{j}-1\right)=n-1 .
$$

Soit:

$$
2 n-(p+q)=n-1 \text { ou encore } p+q=n+1 . \square
$$

\subsection{Assortiment des Degrés d'un Arbre Bicolorié}

Tout arbre a une structure naturelle de graphe bipartite: on peut colorier alternativement les sommets en noir et blanc. Supposons choisi l'un des deux coloriages possibles; soit $n$ le nombre d'arêtes de l'arbre, et soit $\alpha=\alpha_{1}, \alpha_{2}, \ldots, \alpha_{p}$ (resp. $\beta=\beta_{1}, \beta_{2}, \ldots, \beta_{q}$ ) la suite des degrés (ou valences) des sommets noirs (resp. blancs), ordonnée dans le sens décroissant. Comme toute arête joint un sommet blanc et un sommet noir, on a:

$$
\sum_{i=1}^{p} \alpha_{i}=\sum_{j=1}^{q} \beta_{j}=n .
$$

D'autre part l'arbre comporte $p$ sommets noirs, et $q$ sommets blancs, donc $p+q=$ $n+1$. On appellera assortiment de degrés d'un arbre bicolorié le couple $(\alpha, \beta)$, et pour abréger on dira que l'arbre est de type $\left\langle\alpha_{1}, \alpha_{2}, \ldots, \alpha_{p} ; \beta_{1}, \beta_{2}, \ldots, \beta_{q}>\right.$; par exemple l'arbre de la Fig.2 est de type $<321 ; 3111>$. Réciproquement:

Proposition 2. Soient deux partitions de l'entier $n$, avec respectivement $p$ et $q$ parts, telles que $p+q=n+1$; il existe alors au moins un arbre bicolorié admettant ces partitions pour assortiment de degrés.

Preuve (par récurrence): on vérifie facilement que les hypothèses impliquent que, pour $n \geq 2$, on a forcément $\alpha_{1}>1$ et $\beta_{q}=1$ (ou l'inverse); par hypothèse de récurrence, il existe un arbre de type $\left\langle\alpha_{1}-1, \alpha_{2}, \ldots, \alpha_{p} ; \beta_{1}, \beta_{2}, \ldots, \beta_{q-1}\right\rangle$, et il suffit de relier le sommet de degré $\alpha_{1}-1$ à une nouvelle feuille blanche pour obtenir un arbre du type souhaité.

\subsection{Système Associé à un Assortiment de Degrés}

Le calcul du polynôme de Shabat associé à un arbre plan de type

$$
<\alpha_{1}, \alpha_{2}, \ldots, \alpha_{p} ; \beta_{1}, \beta_{2}, \ldots, \beta_{q}>
$$

revient à trouver les valeurs des complexes:

$$
\lambda, a_{1}, a_{2}, \ldots, a_{p}, b_{1}, b_{2}, \ldots, b_{q}
$$

telles que l'on ait simultanément:

$$
A(z)=\lambda\left(z-a_{1}\right)^{\alpha_{1}} \ldots\left(z-a_{p}\right)^{\alpha_{p}}
$$


et:

$$
A(z)-1=\lambda\left(z-b_{1}\right)^{\beta_{1}} \ldots\left(z-b_{q}\right)^{\beta_{q}} .
$$

En outre les valeurs des $a_{i}$ et des $b_{j}$ doivent être toutes distinctes. De façon équivalente, on cherche des polynômes unitaires:

$$
A(z)=\prod_{i=1}^{p}\left(z-a_{i}\right)^{\alpha_{i}}, \quad B(z)=\prod_{j=1}^{q}\left(z-b_{j}\right)^{\beta_{j}}
$$

tels que les polynômes dérivés vérifient $A^{\prime}=B^{\prime}$; en effet on a alors, pour tout $z$ : $A(z)=B(z)-\gamma$, et il suffit de multiplier $A$ et $B$ par $\lambda=1 / \gamma$.

L'équation $A^{\prime}=B^{\prime}$ fournit $n-1$ relations algébriques entre les $p+q=n+1$ inconnues, ce qui est cohérent avec le Théorème 1, qui affirme que le dessin est unique dès qu'on en a fixé deux points. Dans les calculs, il faut cependant veiller à ne pas fixer un sommet "ambigü", c'est à dire non unique parmi les sommets de même couleur et de même degré: sinon le nombre de solutions trouvées est multiplié par le nombre de choix possibles pour lever l'ambiguïté. Cette question est plus délicate qu'il n'y paraît, et nous appellerons célibataire un sommet unique parmi les sommets de même couleur et de même degré.

L'équation $A^{\prime}=B^{\prime}$ peut se simplifier selon une méthode qui nous a été suggérée par H.Cohen, et qui se traduit en quelques lignes de Maple; il existe d'autres façons de procéder (voir [3]). On considère les polynômes $R$ et $S$ qui admettent les mêmes racines que $A$ et $B$, mais avec une multiplicité diminuée de 1:

$$
R(z)=\prod_{i=1}^{p}\left(z-a_{i}\right)^{\alpha_{i}-1}, \quad S(z)=\prod_{j=1}^{q}\left(z-b_{j}\right)^{\beta_{j}-1}
$$

$R$ n'est autre que le PGCD de $A$ et $A^{\prime}$, donc $A^{\prime}=R T$; de même $B^{\prime}=S U$, et l'équation $A^{\prime}=B^{\prime}$ devient: $R T=S U ; R$ et $S$ sont premiers entre eux, car tous les sommets de l'arbre sont distincts; donc $R$ divise $U$ et $S$ divise $T$; l'examen des degrés et des termes de plus haut degré montre qu'en fait:

$$
T=n S, \quad U=n R
$$

ce qui réduit le degré des équations.

Le corps de définition d'un dessin contient les coefficients d'un polynôme de Shabat, et non ses racines; on regroupe donc, au début du calcul, les sormmets de même degré, ce qui revient à écrire:

$$
A=\prod A_{k}^{k}, \quad B=\prod B_{l}^{l}
$$

où chaque $A_{k}$ n'admet que des racines simples (autrement dit est sans carré), et a pour degré le nombre de sommets noirs de valence $k$; le produit porte sur les indices $k$ tels qu'il existe au moins un tel sommet; idem mutatis mutandis pour les $B_{l}$. 


\section{Exemples}

Cette section montre le déroulement des calculs pour des arbres avec 6 arêtes; les sessions Maple ont été éditées pour être plus lisibles. Les inconnues sont les coefficients des polynômes $A_{k}$ et $B_{l}$ définis par (3); leurs noms commencent par $a, b$ ou c, bien qu'elles ne correspondent pas en général aux racines des polynômes $A$ et $B$, contrairement aux notations de la section 3 . Les polynômes dérivés $A^{\prime}$ et $B^{\prime}$ sont notés dA et $\mathrm{dB}$.

\subsection{Type $<411 ; 3111>$}

On vérifie facilement qu'il existe un seul arbre plan combinatoire pour cet assortiment de degrés; d'autre part le sommet noir de degré 4 et le sommet blanc de degré 3 sont célibataires: on peut donc les placer arbitrairement en 0 et 1 respectivement, et le calcul fournira une seule solution, d'après le Théorème 1 .

$>\mathrm{A}$;

$\mathrm{B}$;

$>\mathrm{dA}$;

$$
z^{4}\left(z^{2}+a 1 z+a 0\right)
$$

$$
(z-1)^{3}\left(z^{3}+b 2 z^{2}+b 1 z+b 0\right)
$$

$$
\begin{aligned}
& 4 z^{3}\left(z^{2}+a 1 z+a 0\right)+z^{4}(2 z+a 1) \\
& \quad=z^{3}\left(6 z^{2}+5 a 1 z+4 a 0\right)
\end{aligned}
$$

$>\mathrm{dB}$;

$$
\begin{aligned}
& 3(z-1)^{2}\left(z^{3}+b 2 z^{2}+b 1 z+b 0\right)+(z-1)^{3}\left(3 z^{2}+2 b 2 z+b 1\right) \\
& =(z-1)^{2}\left(6 z^{3}+(-3+5 b 2) z^{2}+(-2 b 2+4 b 1) z-b 1+3 b 0\right)
\end{aligned}
$$

Les factorisations ci-dessus de $A^{\prime}$ et $B^{\prime}$ sont les factorisations $A^{\prime}=R T$ et $B^{\prime}=S U$ de la section 3 ; on a donc les équations (2) $T=6 S$ et $U=6 R$ qui, développées par Maple, donnent:

$>$ equations ();

$$
\begin{gathered}
4 a 0=6 \\
5 a 1=-12 \\
-b 1+3 b 0=0 \\
-2 b 2+4 b 1=0 \\
-3+5 b 2=0
\end{gathered}
$$


Comme prévu la solution est unique, et du coup rationnelle; après résolution:

$>\mathrm{A}$;

$>$ simplify $(A-B)$;

$$
z^{4}\left(z^{2}-12 / 5 z+3 / 2\right)
$$

$$
1 / 10
$$

Il faut donc multiplier $A$ par 10 pour obtenir un polynôme ayant pour valeurs critiques 0 et 1 , ce qui donne

$$
z^{4}\left(10 z^{2}-24 z+15\right)
$$

pour polynôme de Shabat; l'arbre est rationnel, et son dessin canonique porte le numéro 5 sur la Fig.3.

\subsection{Type $\langle 411 ; 2211>$}

Il existe deux arbres plans combinatoires pour cet assortiment de degrés: ce sont les arbres numérotés 1.a et 1.b sur la Fig.1, avec le sommet central colorié en noir; c'est le seul sommet célibataire: on le place en 0 . Voici un extrait de la session Maple:

$$
>\mathrm{A} \text {; }
$$

$>\mathrm{B}$;

$$
z^{4}\left(z^{2}+a 1 z+a 0\right)
$$

$$
\left(z^{2}+b_{1} z+b 0\right)^{2}\left(z^{2}+c 1 z+c 0\right)
$$

$>$ equations ();

$$
\begin{gathered}
4 \mathrm{a} 0=6 \mathrm{~b} 0 \\
5 \mathrm{a} 1=6 \mathrm{~b} 1 \\
2 \mathrm{~b} 1 \mathrm{c} 0+\mathrm{b} 0 \mathrm{c} 1=0 \\
3 \mathrm{~b} 1 \mathrm{c} 1+4 \mathrm{c} 0+2 \mathrm{~b} 0=0 \\
5 \mathrm{c} 1+4 \mathrm{~b} 1=0
\end{gathered}
$$

On calcule $c_{1}$ et $c_{0}$ en fonction de $b_{1}$ et $b_{0}$ (2 dernières équations) puis $b_{1}$ et $b_{0}$ en fonction de $a_{1}$ et $a_{0}$ (2 premières équations); il reste l'équation du milieu, notée $x_{2}=0$ dans la session Maple:

$>$ primpart( $(x 2)$;

$$
25 \text { a1 }-36 \text { a1 a0 }
$$

L'équation $x_{2}=0$ se décompose évidemment: 
1. $a_{1}=0$ : le milieu des feuilles noires est confondu avec le sommet d'ordre 4; il reste le choix d'un paramètre, par exemple $a_{0}=1$.

2. $a_{1} \neq 0$ : on peut alors choisir $a_{1}$, et calculer $a_{0}$; par exemple $a_{1}=6$ entraîne $a_{0}=25$.

Les arbres étudiés sont donc à nouveau rationnels; la Fig. 3 donne leurs dessins canoniques (assortiment de degrés numéro 6).

\subsection{Type $\langle 321 ; 2211>$}

Il existe trois arbres plans combinatoires pour cet assortiment de degrés, parmi lesquels les arbres numérotés $3 . \mathrm{a}$ et 3.b sur la Fig.1. On place respectivement en 0 et 1 les sommets noirs célibataires de degrés 3 et 2 :

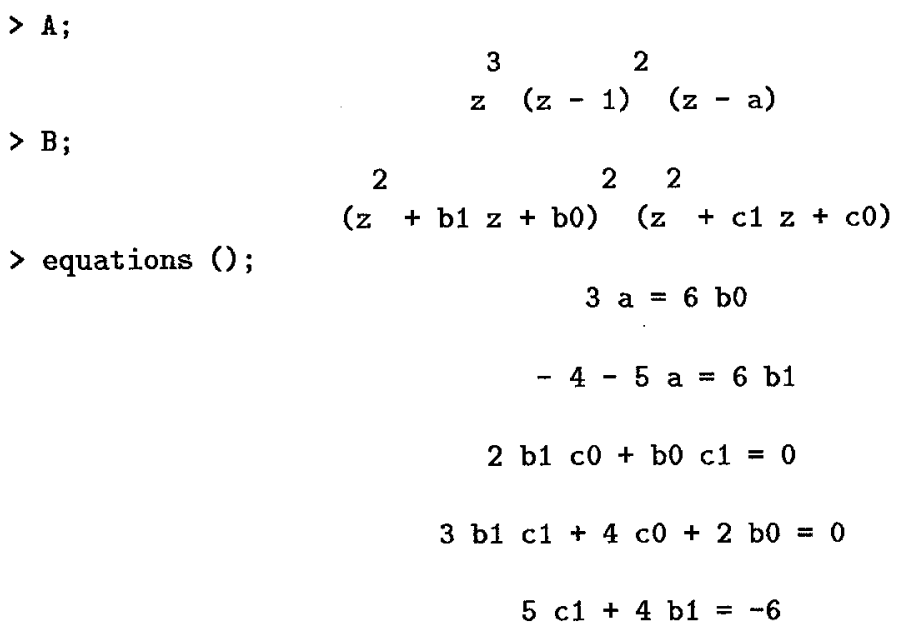

On calcule $c_{1}$ et $c_{0}$ en fonction de $b_{1}$ et $b_{0}$ (2 dernières équations) puis $b_{1}$ et $b_{0}$ en fonction de $a$ ( 2 premières équations); il reste l'équation du milieu, notée à nouveau $x_{2}=0$ :

$>$ primpart $(\mathrm{x} 2)$;

$$
16+24 a+12 a^{2}-25 a^{3}
$$

On vérifie que ce dernier polynôme est irréductible; les trois arbres plans combinatoires de cette classe sont donc conjugués: chacun correspond à une racine de ce polynôme. Les arbres plans 3.a et 3.b de la Fig.1, symétriques l'un de l'autre, correspondent aux deux racines complexes conjuguées; la racine réelle correspond au premier arbre plan de la colonne droite sur la Fig.4; les graphes sous-jacents aux deux derniers dessins de cette colonne sont isomorphes, pas le premier.

Les racines du polynôme $16+24 a+12 a^{2}-25 a^{3}$ s'expriment en fonction de $\sqrt[3]{2}$, et son groupe de Galois est facilement calculable: c'est $S_{3}$ (groupe symétrique d'ordre 3 ), comme c'est le cas le plus souvent pour un polynôme de degré 3 . 
La Fig. 4 montre les deux orbites d'arbres plans conjugués avec 6 arêtes; la première orbite, de taille 2, correspond aux arbres 2.a et 2.b de la Fig.1; les Figs. 3 et $\mathbf{4}$ fournissent donc la liste complète des dessins canoniques d'arbres avec 6 arêtes. Notons que dans le cas d'arbres conjugués, les positions mutuelles sont elles aussi canoniques.

\section{Perspectives}

Les calculs deviennent vite plus difficiles: pour certaines classes d'arbres à 7 ou 8 arêtes, le calcul produit plusieurs équations algébriques, et il faut employer les bases de Gröbner, ou une autre méthode sophistiquée, pour les résoudre. $\mathrm{Au}$-delà, les calculs deviennent souvent impraticables, ce que l'argument suivant explique: les séries énumératrices des arbres plans et des partitions sont bien connues; en les comparant, on constate que le nombre moyen d'arbres plans, pour un assortiment de degrés donné, croît très rapidement: pour 24 arêtes, on peut s'attendre à ce que les coefficients du polynôme de Shabat soient racines d'équations de degré supérieur au million!

Les questions principales de cette théorie concernent le corps de définition d'un arbre, qu'on aimerait relier aux propriétés combinatoires de l'arbre; par exemple;

1. Comment caractériser les arbres rationnels?

2. Comment caractériser les arbres dont le corps de définition est quadratique?

3. Quand et comment une classe d'arbres, pour un assortiment de degrés donné, se scinde-t-elle en plusieurs classes de conjugaison?

4. Comment caractériser les arbres dont le corps de définition est cubique, et dont le groupe de Galois est le groupe cyclique $C_{3}$ (au lieu du groupe symétrique $\left.S_{3}\right)$ ?

On a les réponses partielles suivantes:

Proposition 3. Soit $(\alpha, \beta)$ un assortiment de degrés pour lequel il existe un seul arbre plan combinatoire $\Gamma$; alors $\Gamma$ est rationnel.

L'exemple 4.1 donne une idée de la preuve; des difficultés supplémentaires surgissent si le dessin ne possède pas de sommet célibataire; une preuve rigoureuse se trouve dans [1].

La réciproque est fausse, comme le prouve l'exemple 4.2. Nonobstant, N.Adrianov a réalisé une classification exhaustive des assortiments de degrés pour lesquels il existe un seul arbre plan combinatoire. Les cas les plus simples sont:

1. $\langle n ; 11 \ldots 1\rangle$ : ce cas est celui du polynôme $z^{n}$, et le dessin correspondant possède comme sommets blancs les racines $n^{\text {èmes }}$ de l'unité, reliées à l'origine (sommet noir de degré $n$ ); voir dessin numéro 1, Fig.3.

2. $<22 \ldots 2 ; 22 \ldots 211>$ ou $<22 \ldots 21 ; 22 \ldots 21>$ selon la parité du nombre d'arêtes; l'arbre est une chaîne, et son polynôme de Shabat vaut: $\left(T_{n}+1\right) / 2$ où $T_{n}$ désigne le polynôme de Tchebycheff de degré $n$, dont on 
sait qu'il admet pour seules valeurs critiques -1 et 1 , valeurs entre lesquelles

il oscille à la manière d'une sinusoïde; voir dessin numéro 8, Fig.3.

La classification d'Adrianov comporte six classes d'arbres; vérifier que pour chaque cas il existe un seul dessin plan, à isomorphisme près, est assez direct; prouver que la classification est exhaustive est beaucoup plus difficile.

N.Adrianov et G.Shabat ont aussi montré d'une manière constructive:

Proposition 4. Tout corps quadratique est le corps de définition d'un arbre plan.

La proposition suivante répond partiellement à la question 3 ci-dessus:

Proposition 5. Deux arbres conjugués ont même groupe d'automorphismes (en tant qu'arbres plans).

Cette proposition explique que les deux arbres de l'exemple 4.2 ne soient pas conjugués, car l'un est invariant par rotation d'un demi-tour, et pas l'autre. Mais notre catalogue fournit l'exemple de la classe de l'assortiment de degrés $<421 ; 22111>$, qui comporte 4 arbres plans combinatoires; aucun n'admet d'automorphisme non trivial, et pourtant cette classe se scinde en deux classes de conjugaison d'ordre 2.

[8] contient un théorème d'existence en relation avec la question 4:

Proposition 6. Il existe une classe de trois arbres conjugués admettant $C_{3}$ pour groupe de Galois.

Terminons en signalant une question ouverte de dénombrement: quel est le nombre d'arbres plans combinatoires bicoloriés, connaissant leur assortiment de degrés? Il existe des réponses partielles dans $[1,7]$. Il serait intéressant d'ajouter comme paramètre de l'énumération l'ordre de symétrie de l'arbre, à cause de la Prop.5.

\section{Bibliographie}

1. Adrianov, N., Shabat, G.: Dessins planaires à une face et polynômes avec deux valeurs critiques. Prépublication (en russe)

2. Belyi, G.: On the Galois extensions of the maximal cyclotomic field. Math. USSR Izvestia 14 (1980)

3. Bétréma, J., Péré, D., Zvonkin, A.: Plane trees and their Shabat polynomials. Catalog. Publication du LaBRI 92-75 (1992)

4. Grothendieck, A.: Esquisse d'un programme (1984)

5. Harary, F., Prins, G., Tutte, W.T.: The number of plane trees. Koninklijke Nederlandse Akademie Van Wetenschappen 67 (1964) 319-329.

6. Shabat, G., Voevodsky, V.: Drawing curves over number fields, in The Grothendieck Festschrift, vol.3 (1990) 199-227. Birkhäuser Verlag

7. Tutte, W.T.: The number of plane planted trees with a given partition. Amer. Math. Monthly 71 (1964) 272-277

8. Zvonkin, A.: Shabat polynomials for trees of diameter 4. International Conference on Dessins d'enfant, Luminy, April 19-24, 1993. 


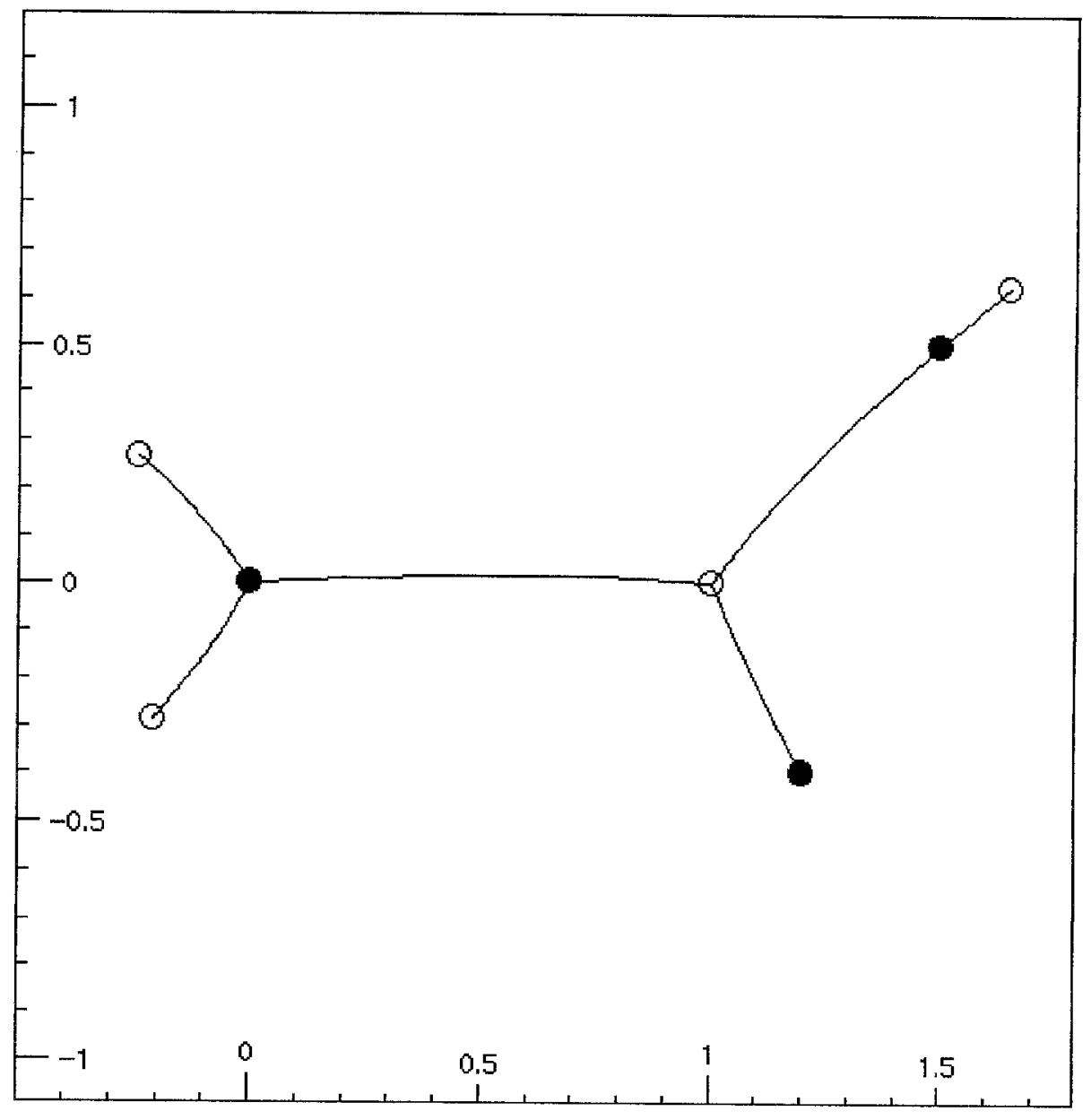

Fig. 2. Dessin canonique de l'arbre 2.a, Fig. 1 
611

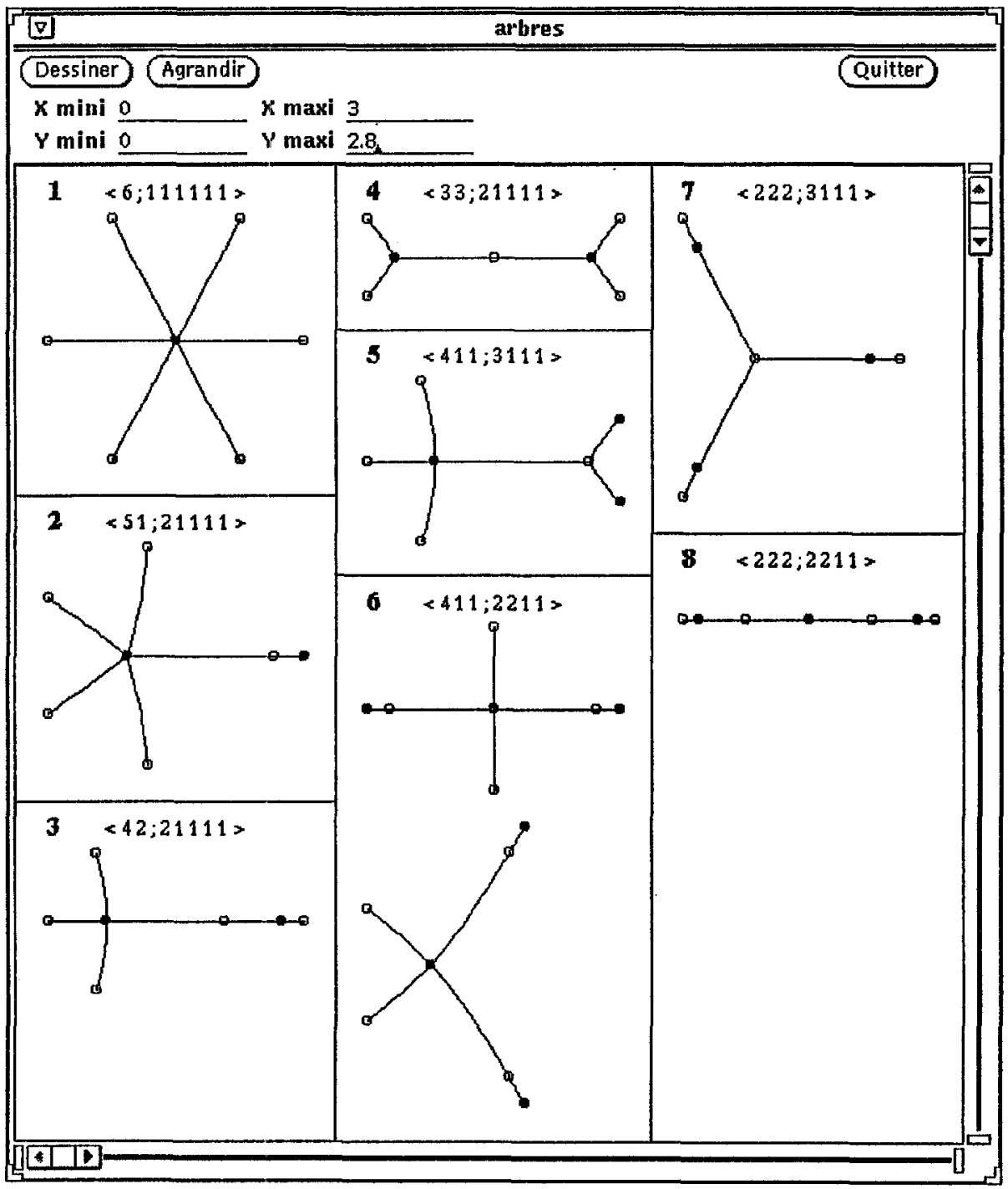

Fig. 3. Arbres rationnels avec 6 arêtes 


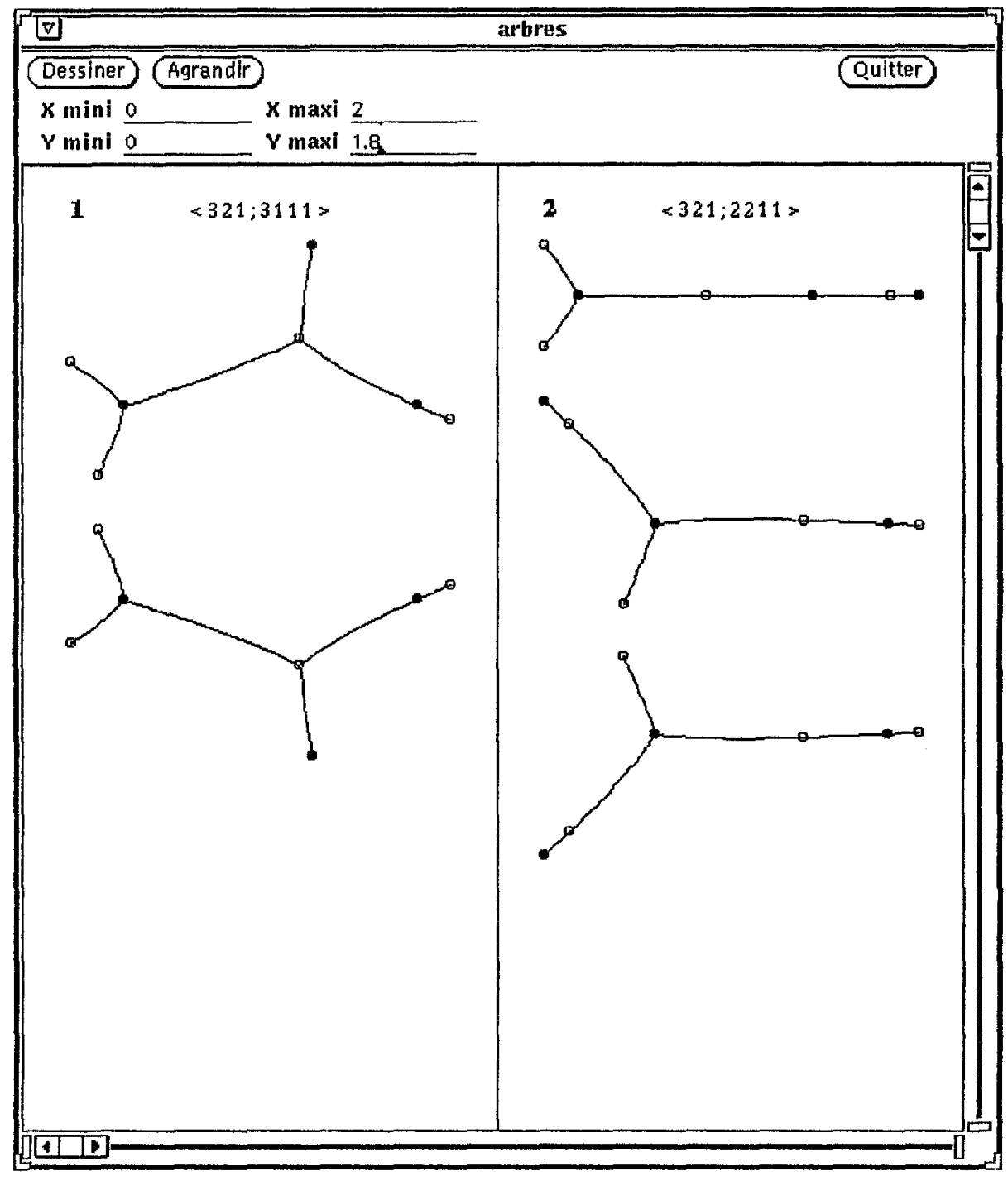

Fig. 4. Arbres conjugués avec 6 arêtes 